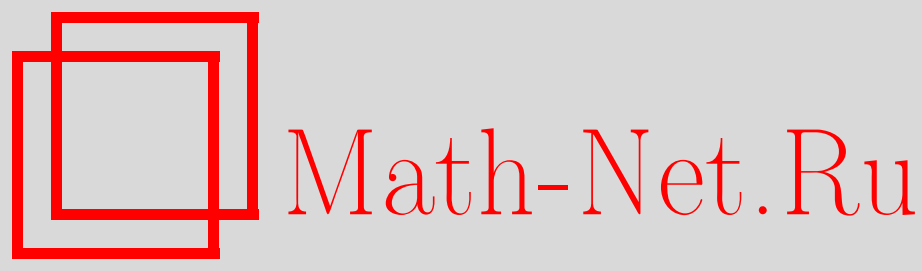

В. В. Мазалов, Л. И. Трухина, Производящие функции и вектор Майерсона в коммуникационных сетях, Дискрет. матем., 2014, том 26, выпуск 3, 65-75

DOI: https://doi.org/10.4213/dm1290

Использование Общероссийского математического портала Math-Net.Ru подразумевает, что вы прочитали и согласны с пользовательским соглашением http://www . mathnet.ru/rus/agreement

Параметры загрузки:

IP : 54.205 .225 .156

26 апреля 2023 г., $17: 56: 55$

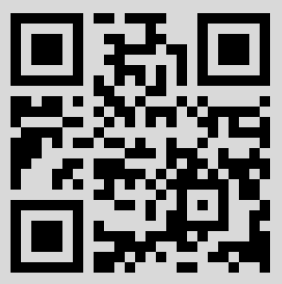




\title{
Производящие функции и вектор Майерсона в коммуникационных сетях
}

\author{
() 2014 г. В. В. Мазалов*, Л. И. Трухина**
}

В работе рассматривается кооперативная игра на неориентированных графах. Характеристическая функция задаётся специальным образом с учётом числа связей и расстояния между вершинами - игроками. Для случая, когда граф является деревом, разработана процедура получения дележа и доказано, что полученный в результате делёж совпадает с вектором Майерсона. Для его вычисления предложено использовать производящую функцию. Показано, что полученные результаты можно обобщить для игры с произвольным графом.

Работа поддержана грантом РФФИ (проект 13-01-91158-ГФЕН) и Отделением математических наук РАН.

Ключевые слова: кооперативные игры, делёж выигрыша, вектор Майерсона.

\section{1. Введение}

Ситуация, в которой любое подмножество множества $N$ игроков может сформировать коалицию и сотрудничать, получая определенные выплаты, может быть описана кооперативной игрой с трансферабельной полезностью (ТП-игрой). Однако существует много реальных ситуаций, для которых требуется модель, принимающая во внимание ограничения в сотрудничестве из-за различных политических, экономических, технических и даже психологических причин (антимонопольное законодательство, отсутствие каналов связи, личные симпатии и антипатии и т.д.) В известной статье Майерсона [1] ограниченная кооперация представлена неориентированным коммуникационным графом. Вершины графа представляют игроков, a рёбра представляют связи между игроками. Игроки могут взаимодействовать напрямую только если они связаны. Понятие связи при этом может интерпретироваться весьма широко: ее наличие может обозначать передачу информации или ресурсов между игроками, отношения сотрудничества и дружбы, наличие транспортной связи. Вершины могут быть отдельными людьми, организациями, странами или веб-страницами. Это приводит к так называемой коммуникационной игре, заданной тройкой, состоящей из конечного множества игроков, характеристической функции и графа. Для такого класса игр Майерсон определил и охарактеризовал

*Место работы: Институт прикладных математических исследований Карельского научного центра РАН, e-mail: vmazalov@krc.karelia.ru

${ }^{* *}$ Место работы: Читинский институт Байкальского государственного университета экономики и права, e-mail: lit-79@mail.ru 
правило распределения, являющееся обобщением вектора Шепли. Впоследствии это правило было названо вектором Майерсона [2].

В 1996 Джексон и Волински [3] предложили модель, названную сетевой игрой, в которой экономические возможности зависят непосредственно от структуры сетей, соединяющих игроков, и обобщили вектор Майерсона в контексте сетевых игр. Несмотря на то, что было предложено и изучено много других правил распределения (см., например, [4] - [10]), вектор Майерсона широко используется в качестве схемы выплаты.

Хотя формула выглядит просто, вычисление вектора Майерсона требует больших усилий. В данной работе для игры со специальной характеристической функцией нами предложена достаточно простая процедура получения дележа, в которой используется производящая функция, и доказано, что полученный в результате делёж совпадает с вектором Майерсона.

\section{2. Основные определения}

Пусть $N=\{1,2, \ldots, n\}-$ множество игроков. Через $2^{N}$ обозначим множество всех его подмножеств.

Кооперативной игрой $n$ лиц будем называть пару $\langle N ; v\rangle$, где $N=\{1,2, \ldots, n\}-$ множество игроков, а $v: 2^{N} \rightarrow R$ отображение, предписывающее каждой коалиции $S \in 2^{N}$ некоторое численное значение, причём $v(\varnothing)=0$. Функция $v$ называется характеристической функцией кооперативной игры [11].

Дележом в кооперативной игре $\langle N ; v\rangle$ называется вектор $x=\left(x_{1}, \ldots, x_{n}\right)$, для которого $\sum_{i \in N} x_{i}=v(N)$ (свойство коллективной разумности), $x_{i} \geqslant v(i), i=1, \ldots, n$, (свойство индивидуальной рациональности).

Неориентированный граф $g=(N, E)$ состоит из множества вершин $N$ и множества рёбер $E$. Рёбра представляют неупорядоченные пары вершин, которые мы будем обозначать $i j$, и $i j \in E$ означает, что вершины $i \in N$ и $j \in N$ связаны в графе $g$.

Вершины графа идентифицируются с игроками, а наличие ребра ij означает, что игроки $i$ и $j$ могут взаимодействовать напрямую, если и только если $i j \in g$.

Для графа $g$ последовательность различных вершин $\left\{i_{1}, \ldots, i_{k}\right\}, k \geqslant 2$, есть путь от $i_{1}$ до $i_{k}$, если $i_{h} i_{h+1} \in g$ для всех $h=1, \ldots, k-1$. Длина пути $l$ - число рёбер в нём, $l=k-1$. Расстоянием между двумя вершинами является длина минимального пути между этими вершинами.

Граф $g$ на множестве игроков $N$ связный, если для любых двух вершин существует путь в $g$ от одной вершины к другой.

Коалиция $S$ связна, если любая пара игроков в $S$ связана путём, состоящим только из игроков данной коалиции.

Любая коалиция $S \subseteq N$ единственным образом разбивается графом $g$ на максимальные связные подкоалиции, называемые компонентами связности. Множество всех связных компонент коалиции $S$ обозначим через $S \mid g$ :

$$
S \mid g=\{\{i \mid i \text { и } j \text { связаны в } S \text { через } g\} \mid j \in S\} .
$$

Совокупность $S \mid g$ можно интерпретировать как набор более мелких коалиций, на которые разбита коалиция $S$, если игроки могут взаимодействовать только в соответствии со связями в графе $g$. 
$N \mid g$ означает множество всех связных компонент в $g$.

Граф, получаемый удалением из существующего графа $g$ связи $i j$, мы будем обозначать $g-i j$, а граф, получаемый добавлением в граф $g$ связи $i j$, обозначим $g+i j$.

Ребро $i j$ является мостом в графе $g$, если $g-i j$ имеет больше компонент, чем $g$.

\section{3. Вектор Майерсона}

Пусть даны кооперативная игра $v$ с множеством игроков $N$ и граф $g$, вершинами которого являются игроки. Для каждого игрока $i$, данного графа $g$ и характеристической функции $v$ вектор Майерсона $Y(v, g)=\left(Y_{1}(v, g), \ldots, Y_{n}(v, g)\right)$ определяется следующими аксиомами:

A1 (Аксиома компонентной эффективности). Если $S$ - связная компонента, то сумма выигрышей игроков коалиции $S$ равна полезности, извлекаемой из игры всей коалицией, т.е

$$
\sum_{i \in S} Y_{i}(v, g)=v(S) \quad \forall S \in N \mid g
$$

A2 (Аксиома справедливости). Для каждого графа $g$, для каждого ребра $i j \in g$ оба игрока одинаково получают выгоду или теряют от создания связи:

$$
Y_{i}(v, g)-Y_{i}(v, g-i j)=Y_{j}(v, g)-Y_{j}(v, g-i j) .
$$

Если для любой коалиции $S$ определить характеристическую функцию как

$$
v_{g}(S)=\sum_{K \in S \mid g} v(K)
$$

то вектор Майерсона может быть вычислен по формуле

$$
Y_{i}(v, g)=\sum_{S \subset N \backslash\{i\}}\left(v_{g}(S \cup i)-v_{g}(S)\right) \frac{s !(n-s-1) !}{n !},
$$

где $s=|S|, \quad n=|N|$.

\section{4. Характеристическая функция}

Рассмотрим игру, в которой граф $g$ является деревом, состоящим из $n$ вершин, а характеристическая функция задаётся подобно схеме, предложенной Джексоном 1 $[3,12]$ : каждая прямая связь - путь длиной 1 - приносит игрокам доход $r$, где $0 \leqslant r \leqslant 1$. Кроме того, игроки также извлекают выгоду из косвенных (непрямых) связей, но уже меньшую. За каждый путь длиной 2 коалиция получает $r^{2}$, за путь длиной 3 получает $r^{3}$ и т. д. Для любой коалиции $S$ можно записать

$$
v(S)=a_{1} r+a_{2} r^{2}+\ldots+a_{L} r^{L}=\sum_{k=1}^{L} a_{k} r^{k},
$$

где $L-$ максимальное расстояние между двумя вершинами в данной коалиции, $a_{k}$ - число путей длины $k$ в данной коалиции;

$$
v(i)=0 \forall i \in N
$$




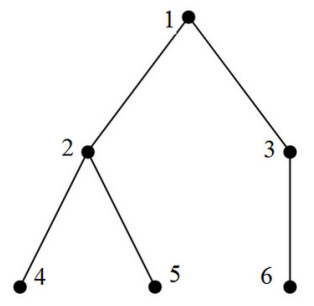

Рис. 1: Дерево 1.

Пример 1. Для дерева на рис. 1 имеем $L=4, a_{1}=5, a_{2}=5, a_{3}=3$, $a_{4}=2$. Характеристическая функция для коалиции, в которую входят все игроки (гранд-коалиции), равна

$$
v(N)=5 r+5 r^{2}+3 r^{3}+2 r^{4} .
$$

Для коалиции $S=\{1,2,4,5\}$ выполняются равенства $L=2, a_{1}=3, a_{2}=3$ и характеристическая функция равна

$$
v(S)=3 r+3 r^{2} .
$$

\section{5. Принцип дележа}

Опишем процедуру получения выигрыша для произвольного игрока $i$.

Шаг 1. Два напрямую связанных игрока получают $r$. По отдельности они не получат ничего, поэтому каждый из них вправе рассчитывать на половину, т.е. выигрыш составит $\frac{r}{2}$. Но если игрок участвует в нескольких таких связях, то он получит $\frac{r}{2}$ от каждой. Значит, его выигрыш нужно умножить на количество путей длины 1 , содержащих этого игрока.

Шаг 2. Чтобы получить $r^{2}$, нужен путь из трёх игроков. Без любого из них коалиция заработает меньше, поэтому каждый из трёх должен получить $\frac{r^{2}}{3}$ от всех путей длины 2 , проходящих через него.

Рассуждая аналогичным образом, и суммируя выигрыши каждого шага, получим делёж:

$$
Y_{i}(v, g)=\frac{A_{1}^{i}}{2} r+\frac{A_{2}^{i}}{3} r^{2}+\ldots+\frac{A_{L}^{i}}{L+1} r^{L}=\sum_{k=1}^{L} \frac{A_{k}^{i}}{k+1} r^{k},
$$

где $A_{k}^{i}$ - число путей длины $k$, содержащих игрока $i$.

Пример 2. Найдём выигрыш игрока 2 из примера 1. Перечислим все пути, содержащие игрока 2. Пути длиной $1:\{1,2\},\{2,4\},\{2,5\}$, т.е. $A_{1}^{2}=3$. Пути длиной 2 : $\{1,2,4\},\{1,2,5\},\{4,2,5\},\{2,1,3\}, A_{2}^{2}=4$. Пути длиной $3:\{3,1,2,4\},\{3,1,2,5\},\{2,1,3,6\}$, $A_{3}^{2}=3$. Пути длиной $4:\{4,2,1,3,6\},\{5,2,1,3,6\}, A_{4}^{2}=2$. Тогда

$$
Y_{2}=\frac{3}{2} r+\frac{4}{3} r^{2}+\frac{3}{4} r^{3}+\frac{2}{5} r^{4}
$$

Покажем, что предложенный нами делёж удовлетворяет аксиомам A1 и А2, и, следовательно, является вектором Майерсона.

\footnotetext{
${ }^{1}$ В модели связей (connections model), введённой Джексоном и Волынски, таким образом задаётся полезность каждого игрока. Кроме того, в модель включена стоимость поддержания связи.
} 
Аксиома А1 выполняется в силу способа задания характеристической функции и дележа.

Покажем, что делёж удовлетворяет аксиоме А2.

Например, если удалить связь между вершинами $i$ и $j$, то получим дерево $g-i j$, состоящее из двух деревьев (рис. 2б).

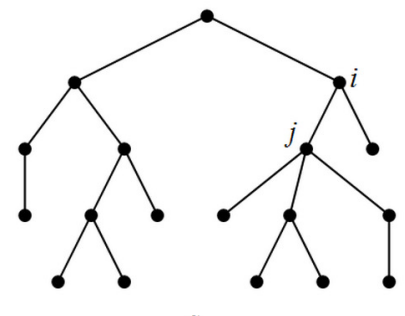

$a$

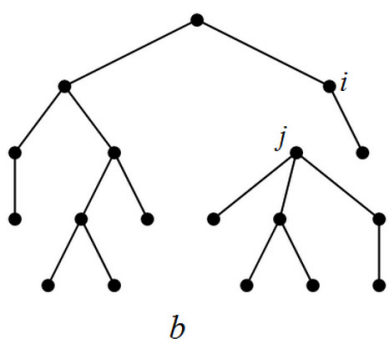

$b$

Рис. 2: а) дерево $g$, б) дерево $g-i j$.

Число путей длины $k$, не содержащих ребро $i j$, после его удаления у обоих игроков не изменится. А число путей длины $k$, проходивших через ребро $i j$, сократится одинаково и для игрока $i$ и для игрока $j$. Так как выигрыши игроков зависят от числа путей, то и изменения в выигрышах будут одинаковыми.

Отсюда следует, что

$$
Y_{i}(v, g)-Y_{i}(v, g-i j)=Y_{j}(v, g)-Y_{j}(v, g-i j)
$$

\section{6. Производящая функция для числа путей}

При большом $N$ практическое применение формулы (5) довольно трудоёмко. Упростить этот процесс можно с помощью производящей функции.

Рассмотрим дерево $g_{p}=(N, E)$ с корнем в вершине $p$. Введём в рассмотрение производящую функцию

$$
\varphi_{p}(x)=\sum_{k=1}^{L} \alpha_{k}^{p} x^{k}
$$

где $\alpha_{k}^{p}$ - число путей, состоящих из $k$ игроков (длины $\left.k-1\right)$, содержащих вершину $p$.

Для нахождения этой функции нами предлагается модификация алгоритма Джемисона [13], который ввёл производящую функцию для числа поддеревьев из $k$ вершин дерева $g$.

Вычислим производящую функцию рекуррентно. Вначале определим её в финальных вершинах $q$ дерева $g_{p}$

$$
\varphi_{q}(x)=x
$$

Обозначим $l$ число игроков на максимальном пути $\{p, \ldots, q\}$. Рассмотрим вершины дерева, для которых число игроков на пути $\{p, \ldots, q\}$ равно $l-1$. Если $q \neq p$, то положим

$$
\varphi_{q}(x)=x\left(1+\sum \varphi_{q_{i}}(x)\right)
$$


где сумма берётся по всем потомкам $q_{i}$ вершины $q$. Затем рассматриваем пути с $l-2$ игроками. Так продолжаем до путей с двумя игроками включительно. Тогда производящая функция будет определена для всех потомков вершины $p$. Теперь положим

$$
\varphi_{p}(x)=x\left(1+\sum \varphi_{q_{i}}(x)+\sum_{i \neq j} \varphi_{q_{i}}(x) \varphi_{q_{j}}(x)\right)
$$

где сумма берётся по всем потомкам $q_{i}$ вершины $p$.

Доказательство основано на следующих соображениях. Пусть $q_{1}, \ldots, q_{d}$ - потомки вершины $q \neq p, d$ - число потомков. Тогда любой путь из $q$ в вершину $s$ в дереве $g_{q}$ проходит через одну из вершин $q_{i}$, при этом разница в длинах пути составляет единицу.

Если же $q=p$, то к путям из $k$ игроков, которые начинаются из вершины $p$, нужно добавить ещё пути из $k$ игроков, которые проходят через $p$ и могут быть составлены из путей, состоящих из $k_{1}<k$ игроков в дереве $g_{q_{i}}$ и путей, состоящих из $k-k_{1}$ игроков в дереве $g_{q_{j}}$, где $i \neq j$. Число таких составных путей и определяется из произведения во второй сумме выражения (7).

Пример 3. Для дерева из примера 1 для первого игрока получим

$$
\begin{aligned}
& \varphi_{4}(x)=\varphi_{5}(x)=\varphi_{6}(x)=x ; \\
& \varphi_{2}(x)=x\left(1+\varphi_{4}(x)+\varphi_{5}(x)\right)=x(1+2 x) ; \\
& \varphi_{3}(x)=x\left(1+\varphi_{6}(x)\right)=x(1+x) ; \\
& \varphi_{1}(x)=x\left(1+\varphi_{2}(x)+\varphi_{3}(x)+\varphi_{2}(x) \varphi_{3}(x)\right)=x+2 x^{2}+4 x^{3}+3 x^{4}+2 x^{5} . \\
& \text { T.e. } A_{1}^{1}=\alpha_{2}^{1}=2 ; \\
& A_{2}^{1}=\alpha_{3}^{1}=4 ; \\
& A_{3}^{1}=\alpha_{4}^{1}=3 ; \\
& A_{4}^{1}=\alpha_{5}^{1}=2 .
\end{aligned}
$$

\section{7. Общий случай}

Рассмотрим игру с произвольным графом $g$, состоящим из $n$ вершин. В отличие от дерева любые две вершины могут быть связаны несколькими путями разной длины. При построении характеристической функции будем учитывать только минимальные пути. А если две вершины связаны несколькими путями одинаковой длины, то учитываются все такие пути, при условии, что между этими вершинами нет другого меньшего пути. Например, для графа, изображённого на рис. 3, характеристическая функция для гранд-коалиции строится следующим образом. Пути длиной 1: $\{1,2\},\{1,3\},\{2,3\},\{2,4\},\{3,4\}$, т.е. $a_{1}=5$. Пути длиной $2:\{1,2,4\},\{1,3,4\}$ связывают 1 и 4, так как между этими вершинами нет прямой связи, то считаем оба пути. Путь $\{1,2,3\}$ не считаем, так как между 1 и 3 есть прямая связь, $\{2,3,4\}$ не считаем, так как 2 и 4 также связаны напрямую. Т.е. $a_{2}=2$. Путь $\{1,2,3,4\}$ не считаем, так как между 1 и 4 есть путь меньшей длины. Получим следующую характеристическую функцию:

$$
v(N)=5 \cdot r+2 \cdot r^{2}
$$




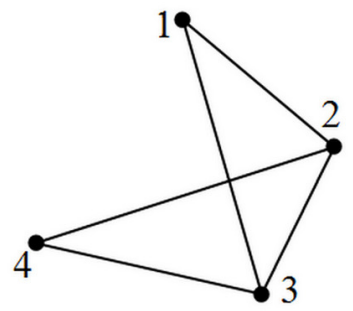

Рис. 3: Произвольный граф.

Для любой коалиции $S$ можно записать

$$
v(S)=a_{1} r+a_{2} r^{2}+\ldots+a_{L} r^{L}=\sum_{k=1}^{L} a_{k} r^{k}
$$

где $L$ - максимальное расстояние между двумя вершинами в данной коалиции; $a_{k}$ - число кратчайших путей длины $k$ в данной коалиции,

$$
v(i)=0, \forall i \in N
$$

Покажем, что и для данной игры описанный выше делёж является вектором Майерсона. Условия аксиомы А1 следуют из определения характеристической функции и дележа .

Аксиома А2.

Пусть граф $h$ получается из графа $g$ удалением связи $i j$, т.е. $h=g-i j$. Если ребро $i j$ является мостом, то при его удалении мы получим граф, состоящий из двух компонент (подграфов). Тогда выполнение аксиомы А2 доказывается аналогично. Если нет, то граф остаётся связным. Пусть длина максимального пути $L$ не изменилась, тогда приращение выигрыша игрока $i$ равно

$$
Y_{i}(v, g)-Y_{i}(v, h)=\sum_{k=1}^{L} \frac{A_{k}^{i}}{k+1} r^{k}-\sum_{k=1}^{L} \frac{B_{k}^{i}}{k+1} r^{k}=\sum_{k=1}^{L} \frac{A_{k}^{i}-B_{k}^{i}}{k+1} r^{k},
$$

где $B_{k}^{i}$ - число путей длины $k$, содержащих игрока $i$, в графе $h$, а приращение выигрыша игрока $j$

$$
Y_{j}(v, g)-Y_{j}(v, h)=\sum_{k=1}^{L} \frac{A_{k}^{j}}{k+1} r^{k}-\sum_{k=1}^{L} \frac{B_{k}^{j}}{k+1} r^{k}=\sum_{k=1}^{L} \frac{A_{k}^{j}-B_{k}^{j}}{k+1} r^{k},
$$

где $B_{k}^{j}$ - число путей длины $k$, содержащих игрока $j$, в графе $h$.

Если путь длины $k$ проходил через ребро $i j$, то при удалении этого ребра число таких путей сократится одинаково и для $i$, и для $j$. Но так как граф связный, то могут добавиться новые пути длиной больше $k$, соединяющие какие-либо две вершины (как минимум это маршруты, соединяющие вершины $i$ и $j$ ). Такие пути должны содержать обе вершины $i$ и $j$, иначе они уже учтены в $A_{k}^{i}$ и $A_{k}^{j}$. То есть числа новых путей и у игрока $i$, и у игрока $j$ должно быть одинаковыми. Из вышесказанного следует, что $A_{k}^{i}-B_{k}^{i}=A_{k}^{j}-B_{k}^{j}$. И окончательно

$$
Y_{i}(v, g)-Y_{i}(v, h)=Y_{j}(v, g)-Y_{j}(v, h) .
$$


Если длина максимального пути уменьшилась или увеличилась, то рассуждения аналогичные.

Обе аксиомы выполняются, следовательно, предложенный делёж является вектором Майерсона и для игры с произвольным графом.

\section{8. Частные случаи}

8.1. Цепь (путь) Пусть граф $g$ - цепь (путь) из $n$ вершин (рис. 4). Максимальное расстояние между двумя вершинами $L=n-1$. Характеристическая функция для гранд-коалиции

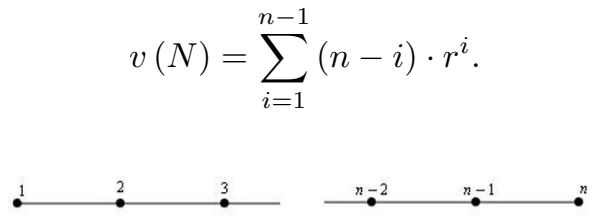

Рис. 4: Цепь (путь).

Для цепи очевидно, что число путей любой длины, содержащих 1-го и $n$-го игроков $A_{k}^{1}=A_{k}^{n}=1$, и для любого игрока число путей максимальной длины $A_{n-1}^{i}=1$.

С учётом этого выигрыши 1-го и $n$-го игроков равны

$$
Y_{1}(v, g)=Y_{n}(v, g)=\frac{1}{2} r+\frac{1}{3} r^{2}+\ldots+\frac{1}{k+1} r^{k}+\ldots+\frac{1}{n} r^{n-1}=\sum_{k=1}^{n-1} \frac{1}{k+1} r^{k} .
$$

Для игроков, занимающих симметричные позиции в цепи, число путей определённой длины, проходящих через них, будет одинаковым. Тода нетрудно показать, что для игроков с номерами $i \in\left\{2, \ldots,\left[\frac{n-1}{2}\right]+1\right\}$

$$
A_{k}^{i}=A_{k}^{n-i+1}=\left\{\begin{array}{rll}
k+1, & \text { если } \quad 1 \leqslant k \leqslant i-1, \\
i, & \text { если } \quad i \leqslant k \leqslant n-i, \\
n-k, & \text { если } \quad n-i+1 \leqslant k \leqslant n-2,
\end{array}\right.
$$

а выигрыш $i$-го игрока

$$
Y_{i}(v, g)=\sum_{k=1}^{i-1} r^{k}+\sum_{k=i}^{n-i} \frac{i}{k+1} r^{k}+\sum_{k=n-i+1}^{n-2} \frac{n-k}{k+1} r^{k}+\frac{1}{n} r^{n-1} .
$$

8.2. Звезда Пусть граф $g$ - звезда из $n$ вершин и игрок 1 - центр звезды (рис. 5). Вычислим вектор Майерсона непосредственно по формуле (3).

Для звезды максимальное расстояние между двумя вершинами $L=2$. Характеристическая функция определяется условиями

$$
\begin{gathered}
v(N)=(n-1) r+C_{n-1}^{2} r^{2}, \\
\forall S \not \supset 1 \quad v(S \cup 1)=s r+C_{s}^{2} r^{2}, \quad v(S)=0 .
\end{gathered}
$$

Найдём выигрыш игрока 1:

$$
Y_{1}(v, g)=\sum_{S \subset N \backslash\{1\}} \frac{s !(n-s-1) !}{n !}\left(v\left(\left.g\right|_{S \cup 1}\right)-v\left(\left.g\right|_{S}\right)\right)=\sum_{S \subset N \backslash\{1\}} \frac{s !(n-s-1) !}{n !} v\left(\left.g\right|_{S \cup 1}\right) .
$$




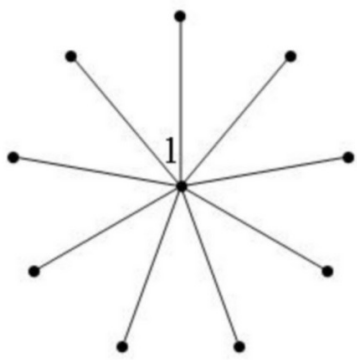

Рис. 5: Звезда.

Выигрыш любой коалиции, содержащей игрока 1, зависит только от количества её членов, а число коалиций из $s$ игроков, не содержащих 1, равно $C_{n-1}^{s}$. Учитывая это, получаем:

$$
\begin{gathered}
Y_{1}(v, g)=\sum_{s=1}^{n-1} \frac{s !(n-s-1) ! C_{n-1}^{s}}{n !}\left(s r+C_{s}^{2} r^{2}\right)= \\
=\frac{1}{n} \sum_{s=1}^{n-1}\left(s r+C_{s}^{2} r^{2}\right)=\frac{1}{n} \sum_{s=1}^{n-1}\left(s r+\frac{s(s-1)}{2} r^{2}\right)= \\
=\frac{1}{n}\left(\frac{(n-1) n}{2} r+\frac{(n-2)(n-1) n}{6} r^{2}\right)=\frac{n-1}{2}\left(r+\frac{n-2}{3} r^{2}\right) .
\end{gathered}
$$

Для остальных игроков

$$
\begin{gathered}
Y_{i}(v, g)=\frac{1}{n-1}\left(v(N)-Y_{1}\right)= \\
=\frac{1}{n-1}\left((n-1) r+\frac{(n-1)(n-2)}{2} r^{2}-\frac{(n-1)}{2} r-\frac{(n-1)(n-2)}{6} r^{2}\right)= \\
=\frac{1}{2} r+\frac{n-2}{3} r^{2}, \quad i=2,3, \ldots, n .
\end{gathered}
$$

Сравним с выигрышами игроков, полученными по формуле (5):

$$
\begin{gathered}
Y_{1}=\frac{A_{1}^{1}}{2} r+\frac{A_{2}^{1}}{3} r^{2}=\frac{n-1}{2} r+\frac{C_{n-1}^{2}}{3} r^{2}= \\
=\frac{n-1}{2} r+\frac{(n-1)(n-2)}{3 \cdot 2} r^{2}=\frac{n-1}{2}\left(r+\frac{n-2}{3} r^{2}\right) . \\
Y_{i}=\frac{A_{1}^{i}}{2} r+\frac{A_{2}^{i}}{3} r^{2}=\frac{1}{2} r+\frac{C_{n-2}^{1}}{3} r^{2}=\frac{1}{2} r+\frac{n-2}{3} r^{2} .
\end{gathered}
$$

Таким образом, выигрыши, вычисленные по формулам (3) и (5), совпадают.

\section{9. Заключение}

В рассматриваемой игре значение вектора Майерсона зависит от числа путей. Следовательно, большее значение выигрыша для игрока означает большее количество путей, проходящих через него, а значит, и больший вес игрока в сети. Поэтому 
можно воспользоваться вектором Майерсона для ранжирования игроков в коммуникационных сетях.

Приведём результаты моделирования для графа, представляющего схему перевозок грузов по Транссибирской магистрали (рис. 6). Вектор Майерсона был вычислен для трёх значений $r=0,9, r=0,5$ и $r=0,2$.

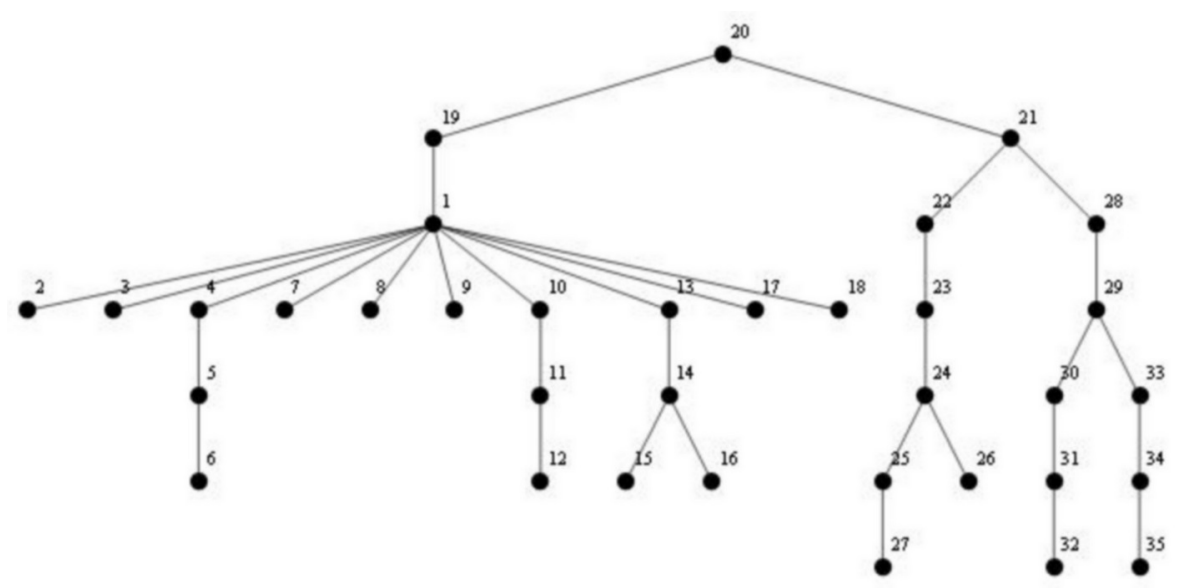

Рис. 6: Граф, построенный на основе схемы перевозки грузов с участием транссибирской магистрали

Москва является самым крупным транспортным узлом, и вершина 1 , соответствующая ей, имеет максимальное значение выигрыша для всех трёх значений $r$. Вершина 21 (Новосибирск) при $r=0,9$ занимает второе место, при $r=0,5$ уступает вершинам 19 и 13 (Екатеринбург и Киев), а при $r=0,2$ только вершине 13. Это означает, что она имеет приоритет по количеству прямых связей и немного уступает по количеству косвенных. Эти города также являются крупными железнодорожными узлами и согласно таблице имеют высокие значения выигрыша. Таким образом, ранжирование, проведённое с помощью вектора Майерсона, достаточно близко к действительности.

\section{Список литературы}

1. Myerson R.B., "Graphs and cooperation in games", Math. Oper. Res., 1977, № 2, 225-229.

2. Aumann R., Myerson R., "Endogenous formation of links between players and coalitions: an application of the Shapley value", The Shapley value, Cambridge University Press, 1988, $175-191$.

3. Jackson M.O., Wolinsky J., "A strategic model of social and economic networks", J. Econ. Theory, 71:1 (1996), 44-74.

4. Borm P., Owen G., Tijs S., "On the position value for communication situations", SIAM J. Discrete Math., 5:3 (1992), 305-320.

5. Borm P., van den Nouweland A., Tijs S., "Cooperation and communication restrictions: a survey", Imperfections and Behavior in Economic Organizations, Kluwer Acad. Publ., Boston, 1994.

6. Calvo E., Lasaga J., van den Nouweland A., "Values of games with probabilistic graphs", Math. Social Sci., 1999, № 37, 79-95.

7. Jackson M.O., "Allocation rules for network games", Games and Econ. Behav., 51:1 (2005), $128-154$. 
8. Slikker M., "Link monotonic allocation schemes", Int. Game Theory Review, 7:4 (2005), 473-489.

9. Slikker M., Gilles R.P., Norde H., Tijs S., "Directed networks, allocation properties and hierarchy formation", Math. Social Sci., 49:1 (2005), 55-80.

10. Talman D., Yamamoto Y., "Average tree solutions and subcore for acyclic graph games", $J$. Oper. Res. Soc. Japan, 51:3 (2008), 187-201.

11. Мазалов В.В., Математическая теория игр и приложения: Учебное пособие, 1-е изд., С-Пб.: Лань, 2010.

12. Jackson M.O., Social and economic networks, Princeton University Press, 2008.

13. Jamison R.E., "Alternating Whitney sums and matchings in trees, part 1", Discrete Math., 1987, № $67,177-189$. 